\title{
Tuning the scattering length on the ground triplet state of $\mathrm{Cs}_{2}$.
}

\author{
V. Kokoouline, J. Vala, and R. Kosloff \\ Fritz Haber Research Center for Molecular Dynamics, Hebrew University of Jerusalem, \\ Jerusalem, 91904, Israel
}

(October 26, 2018)

\begin{abstract}
We develop two schemes for tuning the scattering length on the ground triplet state of $\mathrm{Cs}_{2}$. First, an absolute value of the triplet scattering length of ${ }^{133} \mathrm{Cs}_{2}$ is determined using the experimental data (Fioretti et al, Eur.Phys.J. 5, 389 (1999)). We demonstrate that the large scattering length can be made small and positive by coupling of the ${ }^{3} \Sigma_{u}^{+}(6 S+6 S)$ potential to the ${ }^{3} \Pi_{g}$ state by strong off-resonant radiation. A weaker laser field coupling the ${ }^{3} \Sigma_{u}^{+}(6 S+$ $6 S)$ continuum to the lowest bound level of the excited ${ }^{3} \Sigma_{g}^{+}(6 S+6 P)$ also leads to a small positive scattering length. In addition, the scattering length of the ${ }^{135} \mathrm{Cs}$ isotope is found to be positive. The method used solves the Schrödinger equation for two electronic states coupled by an electromagnetic field with approximations employed. The scattering length is determined from calculated continuum wavefunctions of low energy.
\end{abstract}


A positive scattering length is a crucial ingredient in obtaining Bose-Einstein condensation from a gas of ultra-cold atoms. This parameter controls the long range repulsion between the atoms and therefore the stability of the process. Manipulating the scattering length will allow to add new candidates to the small list of atomic systems which form a condensate. Schemes to control the scattering length have been suggested based on manipulating the inter-atomic interactions by external fields. They include the use of a magnetic field to induce a Feshbach resonance [1] [7, a use of radio-frequency [8] and dc electric fields [9] and off resonant strong electromagnetic field [10 [12]. In the present study we propose two schemes for modifying the scattering length on the ground triplet state of $\mathrm{Cs}_{2}$ using electromagnetic field. First, we calculate the scattering length $a_{s c}$ of triplet ${ }^{133} \mathrm{Cs}_{2}$ and discuss the sensitivity of $a_{s c}$ to the $C_{6}$ coefficient and to the uncertainty of the experimental data. Then, we develop two modification schemes to tune the scattering length to small positive value using continuous wave laser field of experimentally feasible characteristics. In addition, we found that the scattering length of ${ }^{135} \mathrm{Cs}$ is positive and thus this isotope is a suitable candidate for Bose-Einstein condensation of cesium.

The best known potential for the ground triplet state of $\mathrm{Cs}_{2}{ }^{3} \Sigma_{u}^{+}(6 S+6 S)$, is a combination of quantum chemistry calculations by Foucrault et al [13] fitted at the distance $\approx 20$ Bohr to the asymptotic behavior $C_{6} / R^{6}$. In the present calculation we used two different values of the coefficient $C_{6}$ calculated by Marinescu and Dalgarno [14] and by Derevianko et al 15. This potential has $55 \pm 2$ bound vibrational levels. The uncertainty of the number is due to the uncertainty of the potential curves at small distances. Other potentials used for triplet $\mathrm{Cs}_{2}$ [16] lead to a different number of bound vibrational levels, $58 \pm 2$. We checked the influence of the higher order dispersion terms of the long range potential on the results of the calculations. Since no significant differences have been found, we kept only the term $C_{6} / R^{6}$ as a representant of the asymptotic behavior. Using this potential all the bound state wave functions and the threshold scattering wave functions were calculated using the Mapped Fourier Grid Method [17 [19]. This approach enable us to obtain an exact and fully-quantum time-independent solution of the Schrödinger equation without applying 
any approximation. This represent an original and direct approach to the determination of the scattering length. A grid of 557 points covering 20000 Bohr was used. Due to the exponential uniform convergence of the method we were able to converge the error in phase of the wave functions to an accuracy of $\sim 10^{-6}$. At the far end of the grid we used either fixed or absorbing boundary conditions. We found that as expected the threshold continuum wave functions are not affected by the choice of boundary conditions. The accuracy of the calculation is determined by the short-range part of the potential where the inaccuracy of the depth of the well is estimated to be about $40 \mathrm{~cm}^{-1}$ [13. This inaccuracy leads to an asymptotic accumulated phase of more than $\pm 2 \pi$ thus the scattering length cannot be determined from ab initio calculations.

A binary scattering event is completely determined by the inter-atomic potential and the boundary conditions. Since the scattering length is determined by the conditions of vanishing asymptotic kinetic energy it is solea determined by the potential. For heavy colliders such as $\mathrm{Cs}_{2}$ the scattering length is an extremely sensitive function of the potential. Its value is related to the accumulated phase $\phi$ of the wave function from the inner turning point at zero energy to the infinity. The phase $\phi$ for the zero energy is estimated semiclassically as $\int_{R_{i}}^{\infty} \sqrt{-2 \mu U(R)} d R$ where $R_{i}$ is the inner turning point of the potential, $U(R)$ is the potential and $\mu$ is the reduced mass of ${ }^{133} \mathrm{Cs}_{2}$. For the potential used for $\mathrm{Cs}_{2}, \phi$ is $54.6 \pi$. From the Bohr-Sommerfeld quantization condition the number of bound levels should be 54. The number of levels is obtained by a direct solution of the Schrödinger equation is the same. As a consequence, variations of $\pm \pi / 2$ will change the scattering length from negative to positive. These facts pose a computation challenge for ab-initio determination of the scattering length but they also offer an opportunity for experimental manipulation.

To overcome this problem an independent method of determining the phase of the wave function has to be found. The photoassociation spectrum from the triplet to the $0_{g}^{-}(6 S+$ $\left.6 P_{3 / 2}\right)$ state obtained by Fioretti et al 20,21 is used to obtain the phase and to adjust the ground state potential. The spectrum shows oscillating behavior which reflects the variation of the Franck-Condon factors of the transition between the ${ }^{3} \Sigma_{u}^{+}(6 S+6 S)$ and 
rovibrational levels of the $0_{g}^{-}\left(6 S+6 P_{3 / 2}\right)$ state. The oscillations in the spectrum reflect the nodal structure of the continuum wave functions in the energy range of $(200 \mu \mathrm{K})$ [21]. Using the Mapped Fourier Grid Method [17 19], the Franck-Condon factors between the vibrational wave functions of the $0_{g}^{-}\left(6 S+6 P_{3 / 2}\right)$ state and the continuum wave functions of the ${ }^{3} \Sigma_{u}^{+}(6 S+6 S)$ state were calculated. These factors give relative intensities of the spectral lines of the $0_{g}^{-}\left(6 S+6 P_{3 / 2}\right)$ spectrum. The excited potential curve $0_{g}^{-}\left(6 S+6 P_{3 / 2}\right)$ for short distances was obtained by a diagonalization of the ${ }^{3} \Sigma_{g}^{+}(6 S+6 P)$ and ${ }^{3} \Pi_{g}^{+}(6 S+6 P)$ curves of the $0_{g}^{-}(6 S+6 P)$ symmetry while accounting of the spin-orbit interaction $\left(V_{s o}(C s)=554.1\right.$ $\left.\mathrm{cm}^{-1}\right)$. In the asymptotic region the potential is fitted to the RKR-potential obtained from the experiment [21].

The inaccuracy of potential leads to a shift of the position of nodes of the calculated spectrum. Fig. (11) shows the calculated intensities (full line) and positions of nodes observed in the experiment. The calculated spectrum is almost completely out of phase with the experiment. By slightly modifying the inner part of the potential within the estimated range of accuracy, the experimental observations can be reconstructed. The variation in the potential can either add or subtract to the phase of the asymptotic part of the wave function. In both cases, we obtained very good agreement with the experimental nodal structure as illustrated in figure 1. With this adjustment the number of bond levels is either 54 or 55 .

In order to see the sensitivity to the inaccuracy of the $C_{6}$ coefficient we used two different values of $C_{6}$ calculated by Marinescu and Dalgarno [14] $\left(C_{6}=-6331\right.$ a.u. $)$ and by Derevianko et al 15 ( $C_{6}=-6899$ a.u.). As a result the inner part of the fitted potential is slightly different. The figure 1 shows the fits for $C_{6}=-6899$ a.u.

Using the adjusted potential which fits the experiment the scattering length is recalculated (Fig. 2). We obtained the value $a_{s c}=-350$ Bohr for $C_{6}=-6331$ and $a_{s c}=-950$ Bohr for $C_{6}=-6899$. For $C_{6}=-6899$ we made additional calculation in order to see if some inaccuracies could lead to the positive scattering length. First we consider the uncertainty of the position of minima in the experimental spectrum. The fitted potential is changed in such a manner that the calculated minimum prior to the last minimum (the last 
node is not very pronounced) shifts by $0.2 \mathrm{~cm}^{-1}$ in two directions. At this condition the last nodes shifts by $0.1 \mathrm{~cm}^{-1}$. The scattering length becomes $a_{s c}=-1040$ Bohr when the nodes shift to the direction of increasing of the binding energy and $a_{s c}=-850$ Bohr for the opposite direction.

We verified also the sensitivity of determination of $a_{s c}$ to the different number of bound levels. With this goal we calculated $a_{s c}$ for the potentials giving 54 and 55 bound levels (dotted and dashed lines on the inset of Fig. 1 correspondingly). We found that once the two potentials are fitted to the same nodal positions, the difference in $a_{s c}$ for the two potentials is smaller at least by a factor 10 than the difference due to the uncertainty of the positions of minima. This results is easily explained in a spirit of the quantum defect theory: If the long-range behavior of the potential and positions of last nodes of the last bound wave function are fixed, the short range dynamics (in particular - the total number of nodes of the wave function at small distances) does not influence the scattering properties.

The obtained value of $a_{s c}$ is in a good agreement with the previous experimental and theoretical results. Arndt et al [22] and Leo et al [23] got the absolute value $>260$ Bohr and $>600$ Bohr respectively. Kokkelmans et al [16] published the value -315 Bohr to -380 Bohr and Legere et al [24] $\approx-400$ Bohr. Recently, Drag et al [25] analyzed the experimental energy of the last bound vibrational levels and determined the triplet state scattering length of cesium from the extrapolated position of the outermost nodes of the bound vibrational wave function [26] obtaining the value ranging from -370 Bohr down to -825 Bohr. The value of $C_{6}=-6510$ was used in their calculations. In addition, we determined the critical $C_{6}$ coefficient for which the scattering length becomes infinite. We found that for $C_{6}=-7000$ it is still negative, but $C_{6}=-7050$ is the transition value. For $C_{6}=-7100$ the scattering length becomes positive. We emphasize the comparison of the presented results with those of Drag et al [25], Tab. [, since both approaches analyze the same set of experimental data by different methods.

The determined absolute value of the scattering length is so large that small variations of the potential can lead to the change of the sign of $a_{s c}$. For example, a low-intensity laser field 
coupling the ground molecular state with one of excited states at large distances can modify the scattering length. This possibility was discussed by Kagan et al [12]. This sensitivity of the sign of $a_{s c}$ to small perturbations has a crucial importance for the character of behavior at ultra cold temperatures. Below we discuss how $a_{s c}$ can be made small and positive.

The sensitivity of the asymptotic phase to the inner part of the potential is the key to modifying the scattering length. The first scheme employs a CW laser field to couple the inner part of the ${ }^{3} \Sigma_{u}^{+}(6 S+6 S)$ potential to the ${ }^{3} \Pi_{g}(6 S+6 P)$ electronic state. The bound and continuum levels are recalculated on the coupled two surface potentials. The rotating wave approximation was used to describe the coupling. The ${ }^{3} \Pi_{g}(6 S+6 P)$ potential is taken from [27].

In order to change the scattering length sufficiently, the adiabatic transition probability, $P\left({ }^{3} \Sigma_{u}^{+} \rightarrow^{3} \Pi_{g}\right)$, for levels with energy close to the $6 S+6 S$ threshold, should be nonnegligible. For relatively small laser intensity this condition is fulfilled only if the potentials ${ }^{3} \Sigma_{u}^{+}(6 S+6 S)$ and ${ }^{3} \Pi_{g}(6 S+6 P)$ cross at an energy close to the $6 S+6 S$ dissociation limit. The insert in figure 3 shows the total two-channel potential in the adiabatic representation. The ${ }^{3} \Pi_{g}(6 S+6 P)$ potential is shifted down by the energy, $E_{f}=\hbar \omega$, determined by the frequency, $\omega$, of the field. When the intensity, $I$, of the laser field is low, the region of a pseudo-crossing is very narrow. Figure 3 shows two wave functions obtained for the two different intensities for the potentials shown at the inset. We found that with increasing field intensity the value of the scattering length gradually moves from negative to positive. A change of sign is obtained for the intensity of $300 \mathrm{~kW} / \mathrm{cm}^{2}$.

The second scheme, is based on a resonant coupling of the continuum wave functions of the ${ }^{3} \Sigma_{u}^{+}(6 S+6 S)$ state to one of vibrational levels of the ${ }^{3} \Sigma_{g}^{+}(6 S+6 P)$ electronic excited state published in 27]. Such a Feshbach resonance results in modification of the triplet state scattering length. The sign and value of the scattering length depends on the position of such a level in respect to the $6 S+6 S$ threshold.

Employing the Mapped Fourier Grid method, the low energy continuum wave functions are calculated for two coupled potentials for different intensities of the laser field. The 
Feshbach resonance is induced by the lowest vibrational level of the excited state negatively detuned from the ground state dissociation threshold by the energy $\Delta=90 \mathrm{MHz}$. From the obtained wave functions the scattering length is determined. A gradual increase of the value of the scattering length is observed reaching high positive values. Since the position (and a width) of the Feshbach resonance depends strongly on the coupling, the scattering length can be changed employing a much smaller field intensity compared to the off-resonant laser manipulation (scheme I). The scattering length could be modified from -350 Bohr to 300 Bohr using a field intensity of only $2.5 \mathrm{~kW} / \mathrm{cm}^{2}$. The projected population in the excited state is less than $10^{-3}$.

We emphasize that the present method of solution of the Schödinger equation on the electronic states coupled by a field does not rely on any approximative treatment like perturbation theory and is therefore exact within a given physical model. That contrasts with the previous approaches by Fedichev et al [10] and Bohn [11].

It is also advantageous to tune the light to one of lowest vibrational levels of the whole $6 S+6 P$ manifold which have allowed dipole transition. In this way one can eliminate the coupling with other vibrational levels of other molecular potentials. This choice of a vibrational level for the Feshbach resonance ensures that a two-photon excitation process to the $(6 P+6 P)$ manifold is avoided. The coupling is also chosen to occur in the short-range region where only a very small fraction of the density resides. This will further reduced loss processes.

The proposed scattering length manipulation techniques employs light induced a coupling of the short-range part of molecular electronic potential. Therefore, we have to consider the variation of the dipole moment due to the molecular orientation with respect to the axis of the field vector. We suggest to solve this problem by using three perpendicular laser beams with perpendicular polarizations for irradiation of the cold cesium sample, which prevents any interference effect. In this setup, we can assume the maximal variation of the dipole moment projection on the field by $\approx 15 \%$. We studied the robustness of the positivity of the scattering length by computing its value for both proposed schemes using 
field intensity varying by several orders of magnitude. We found that both schemes are sufficiently robust to keep the inter-atomic interaction repulsive and, hence, to allow the Bose-Einstein condensation.

Using the adjusted potential the scattering properties of the isotopes ${ }^{135} \mathrm{Cs}$ and ${ }^{137} \mathrm{Cs}$ were calculated (half-life's are $2.3 \cdot 10^{6}$ and 30.2 years respectively). The scattering length of $a_{s c}=165$ Bohr for ${ }^{135} \mathrm{Cs}$ was obtained. This result is in good agreement with the value 138 Bohr published by 16. Therefore, the ${ }^{135} \mathrm{Cs}$ isotope is a good candidate for Bose-Einstein condensation. For the ${ }^{137} \mathrm{Cs}$, we found almost zero scattering length suggesting that this radioactive isotope can behave like a non-interacting Bose gas. In both cases, we tested the value of the scattering length with respect to the number of bound levels of the original fitted potential used. We found no significant difference; specifically, $\approx 7$ Bohr for ${ }^{135}$ Cs $(55$ and 56 bound levels) and $\approx 15$ Bohr for the ${ }^{137}$ Cs isotope (56 and 57 bound levels).

The spontaneous emission broadening of the Feshbach resonance discussed by [10,11] is eliminated in the present scheme in two different ways. First, the population transfer at the short range part of the potential where the resonance is induced is very low due to the small amplitude of the ground state continuum wave function. That results in a small value of the transition dipole moment between both electronic states. Since its order of magnitude is $10^{-4}$, the resulting leading term in the spontaneous emission rate $\left|\left\langle\psi_{g}|\mu| \psi_{e}\right\rangle\right|^{2}$ is approximately $10^{-8}$ extending the spontaneous emission time-scale to values beyond the microsecond range. That reduces the spontaneous emission broadening of the resonance width as well as it eliminates the related leakage mechanism competing with the BoseEinstein condensation. Besides, in agreement with Bohn [11], the coupling is mediated by the field whose intensity, the Rabi frequency, results in the time-scale much shorter, approximately $150 \mathrm{ps}$, than the time-scale of the spontaneous emission and, hence, the spontaneous emission broadening of the Feshbach resonance is negligible compared to its field induced width.

The manipulation of the scattering properties can be checked in other applications. For example photoassociation spectroscopy [28] can be carried out using cold alkali atoms with 
modified scattering properties. The correspondence between the scattering length and the nodal structure of the Franck-Condon factors suggests a direct experimental test of the proposed schemes employing a CW field with intensities obtainable by a diode laser radiation source. The technique can be used to modify existing experiments done with Rb [29] or Cs [20,21].

The change of the scattering length from negative to positive results in decreasing the population density at short inter-nuclear distance by several orders of magnitude. This will decrease the probability for cold molecule formation mediated by three body collisions [30]. As a result the reduction in the Franck-Condon overlap and transition dipole matrix elements will decrease the spontaneous decay loss in the cold molecule formation via photoassociation $20,29,31,32$.

Recently, Söding et al [33 measured very high inelastic collision rates of the spin flip of ground state cesium atoms which results from the negative scattering length. The attractive interaction enhances the ground state population in the inner part of the potential ruling out the Bose-Einstein condensation. Our calculations show that the positive scattering length due to the proposed manipulation schemes will reduce the short range population at least by a factor of 30 . This decreases the spin flip scattering rate almost by three orders of magnitude removing another obstacle to the the Bose-Einstein condensation in cesium.

In conclusion, the present calculation reproduces the oscillations in the $0_{g}^{-}\left(6 S+6 P_{3 / 2}\right)$ photoassociation spectrum of ${ }^{133} \mathrm{Cs}$ by a small adjustment to the ${ }^{3} \Sigma_{u}^{+}(6 S+6 S)$ potential. On this potential we were able to calculate the scattering length of the ${ }^{3} \Sigma_{u}^{+}(6 S+6 S)$ state finding a a value -350 Bohr. Three different schemes to change the scattering length from negative to positive were studied. The scattering length can be modified by a laser field either off-resonant with the $6 S \rightarrow 6 P$ transition or on resonance with one of lowest vibrational levels of $6 S+6 P$ potentials. The third scheme consists in replacing the ${ }^{133} \mathrm{Cs}$ by the ${ }^{135} \mathrm{Cs}$ isotope. For this case the scattering length is 165 Bohr. We also found that another obstacle to the Bose-Einstein condensation, the large rate of inelastic collisions, can be overcome by tuning the scattering length. 
We are grateful to A. Fioretti for many fruitful discussions. The work was supported by the Israel Science Foundation (Moked). The Fritz Haber Research Center is supported by the Minerva Gesellschaft für die Forschung, GmbH München, FRG. 


\section{REFERENCES}

[1] E. Tiesinga et al., Phys. Rev. A 46, R1167 (1992).

[2] E. Tiesinga, B.J. Verhaar, and H.T.C. Stoof, Phys. Rev. A 47, 4114 (1993).

[3] J.M. Vogels et al., Phys. Rev. A 56, R1067 (1997).

[4] S. Inouye et al., Nature (London) 392,151 (1998).

[5] Ph. Courteille et al., Phys. Rev. Lett. 81, 69 (1998).

[6] V. Vuletic et al., Phys. Rev. Lett. 82, 1406 (1999).

[7] V. Vuletic et al., Phys. Rev. Lett. 83, 943 (1999).

[8] A.J. Moerdijk, B.J. Verhaar, and T.M. Nagtegaal, Phys. Rev. A 53, 4343 (1996).

[9] M. Marinescu and L. You, Phys. Rev. Lett. 81, 4596 (1998).

[10] P.O. Fedichev et al., Phys. Rev. Lett. 77, 2913 (1996).

[11] J.L. Bohn and P.S. Julienne, Phys. Rev. A 56, 1486 (1997).

[12] Yu. Kagan, E.L. Surkov, and G.V. Schlyapnikov, Phys. Rev. Lett. 79, 2604 (1997).

[13] M. Foucrault, P. Millié, and J.P. Daudey, J. Chem. Phys. 96, 1257 (1992).

[14] M. Marinescu and A. Dalgarno, Phys. Rev. A 52311 (1995).

[15] A. Derevianko, W.R. Johnson, M.S. Safronova, and J.F. Babb, Phys. Rev. Lett. 82, 3589 (1999).

[16] S.J.J.M.F. Kokkelmans, B.J. Verhaar, and K. Gibble Phys. Rev. Lett. 81, 951 (1998).

[17] R. Kosloff, J. Phys. Chem. 92, 2087 (1988).

[18] V. Kokoouline et al., J. Chem. Phys. 110, 9865 (1999).

[19] V. Kokoouline et al., Phys. Rev. A (August 2000). 
[20] A. Fioretti et al., Phys. Rev. Lett. 80, 4402 (1998).

[21] A. Fioretti et al., Eur. Phys. J. D 5, 389 (1999).

[22] M. Arndt et al., Phys. Rev. Lett. 79, 625 (1997).

[23] P.J. Leo et al., Phys. Rev. Lett. 81, 1389 (1998).

[24] R. Legere and K. Gibble, Phys. Rev. Lett. 81, 5780 (1998).

[25] C. Drag et al., Phys. Rev. Lett. 85, 1408 (2000).

[26] A. Crubellier et al., Eur. Phys. J. D 6 211, (1999).

[27] W. Meyer, (Private communication); N. Spiess, Ph. D thesis, Fachbereich Chemie, Universität Kaiserslautern, (1989).

[28] W.C. Stwalley and H. Wang, J. Mol. Spectr. 195, 194 (1999).

[29] C. Gabbanini et al., Phys. Rev. Lett. 84, 2814 (2000).

[30] T. Takekoshi, B.M. Patterson, and R.J. Knize, Phys. Rev. Lett. 81, 5105 (1998).

[31] N. Nikolov et al., Phys. Rev. Lett., 82, 703 (1999).

[32] N. Nikolov et al., Phys. Rev. Lett., 84, 246 (2000).

[33] J. Söding et al., Phys. Rev. Lett. 80, 1869 (1998). 


\section{TABLES}

TABLE I. The scattering length determination.

\begin{tabular}{|c|c|c|}
\hline \hline & $C_{6}$ & $a_{s c}$ \\
\hline \hline the present work & -6331 & $\approx-350$ \\
\hline Ref. [25] & -6510 & -370 to -825 \\
\hline the present work & -6899 & $\approx-950$ \\
\hline the present work & -7050 & the transition \\
\hline
\end{tabular}




\section{FIGURES}

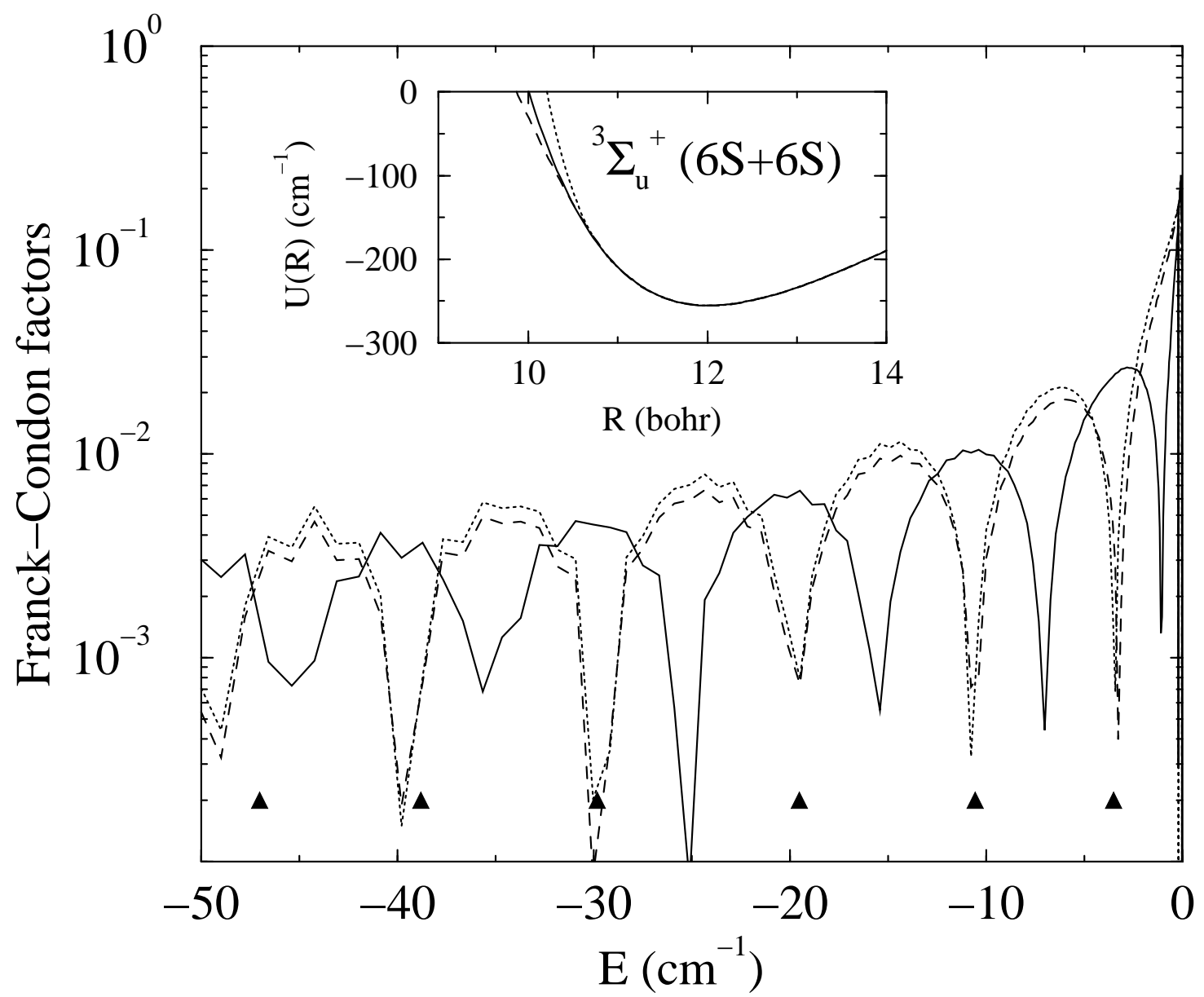

FIG. 1. The variation of the Franck-Condon factors for the transition between the ${ }^{3} \Sigma_{u}^{+}(6 S+6 S)$ and the $0_{g}^{-}\left(6 S+6 P_{3 / 2}\right)$ states for the original (full line) and modified potentials (dotted and dashed lines) with the detuning $E$. Triangles indicate position of the experimental spectral nodes. Inset shows also the original (full line) potential and two modified potentials (dotted and dashed lines) which both reproduce positions of nodes observed experimentally. 


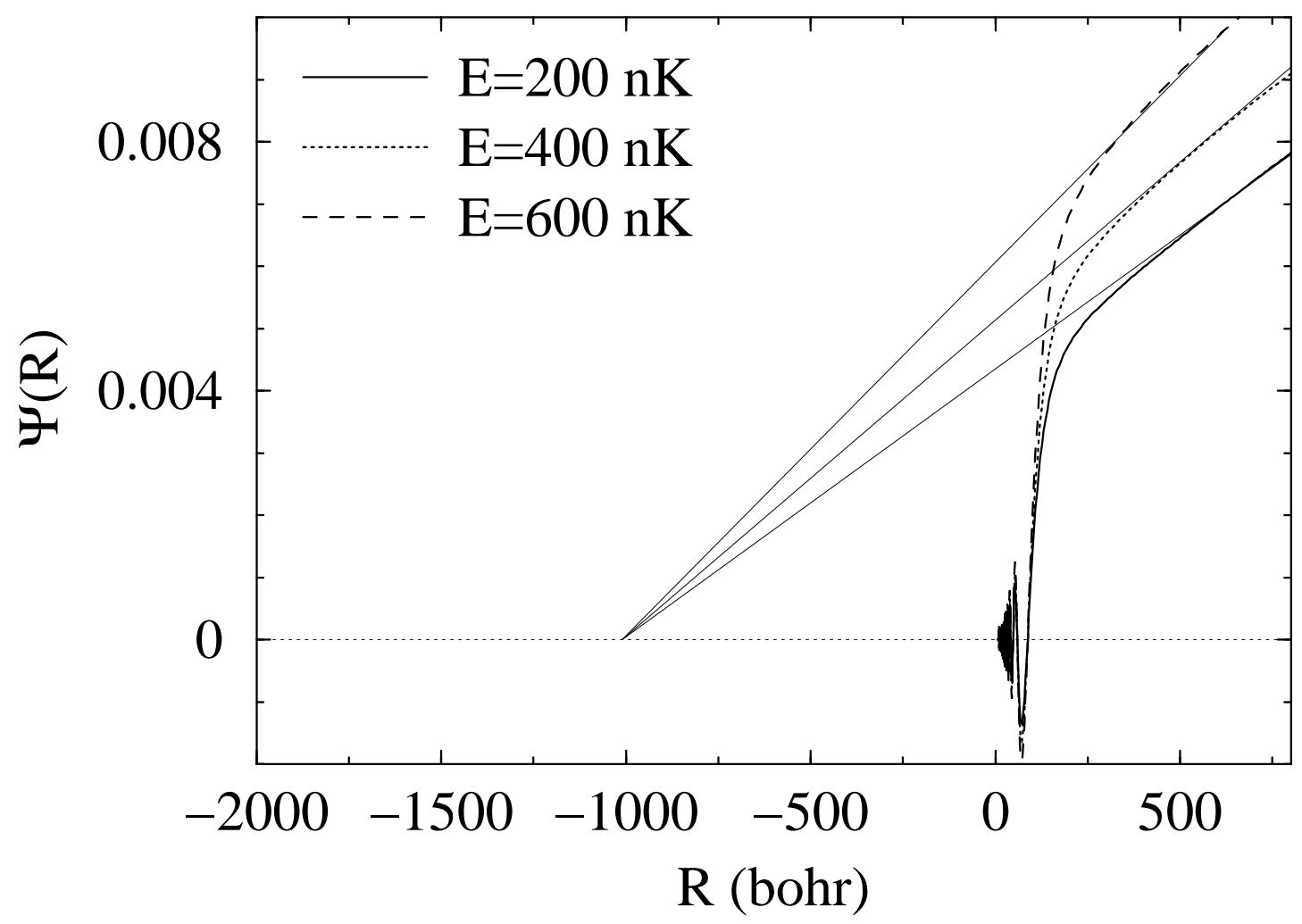

FIG. 2. The determination of the ${ }^{3} \Sigma_{u}^{+}$scattering length from the continuum wave functions slightly above the dissociation limit. The wave functions are calculated using the potential adjusted to reproduce correctly the oscillations in the photoassociation spectrum. 


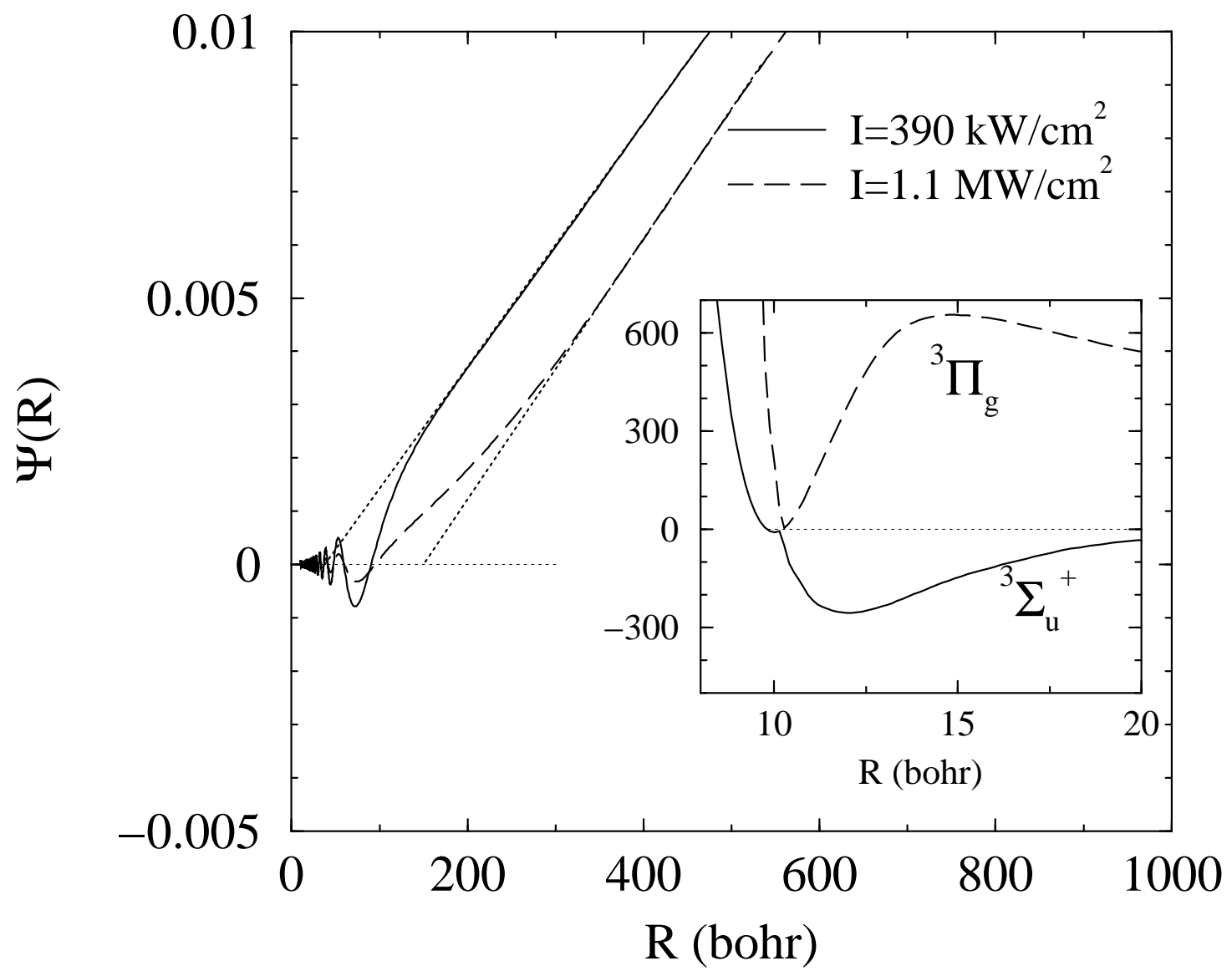

FIG. 3. The scheme of the modification of the inner part of the ${ }^{3} \Sigma_{u}^{+}(6 S+6 S$ ) potential (the inset). The two coupled potentials $\left(\right.$ in $\mathrm{cm}^{-1}$ ) are shown in the adiabatic representation using the rotating wave approximation. Wave functions with energy $E=0.4 \mu \mathrm{K}$, calculated for two different intensities of the laser field. Scattering lengths are 30 Bohr for a intensity $I=390 \mathrm{~kW}$ and 150 Bohr for $I=1.1 \mathrm{MW}$. The energy shift $E_{f}=\hbar \omega$ is the same for both cases, $E_{f}=11408 \mathrm{~cm}^{-1}$ by $324 \mathrm{~cm}^{-1}$ red-detuned to the $6 S \rightarrow 6 P_{3 / 2}$ transition. 


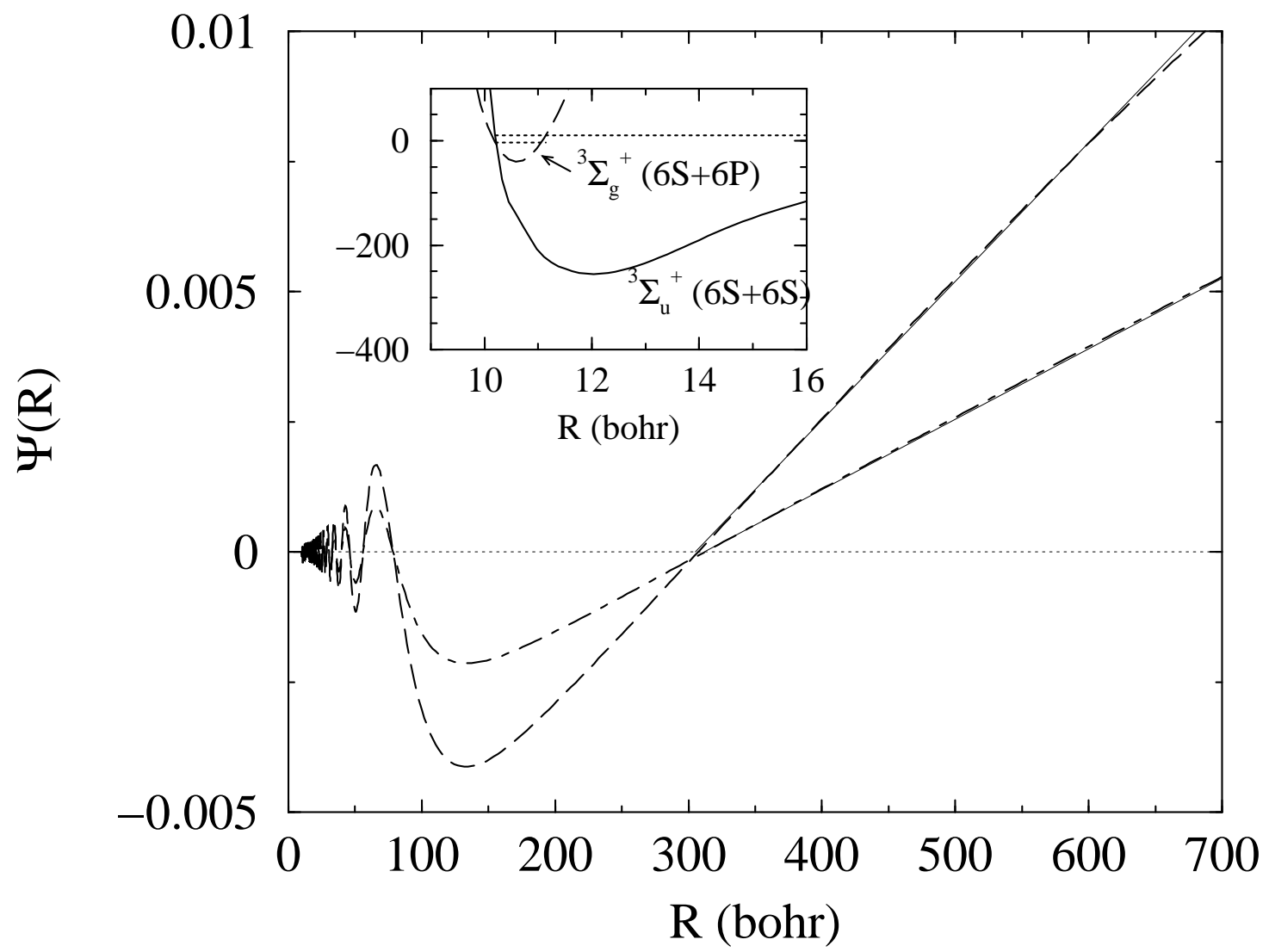

FIG. 4. The continuum wave functions with the scattering length of 300 Bohr obtained by a resonant coupling of a vibrational level in the ${ }^{3} \Sigma_{g}^{+}(6 S+6 P)$ state to the ${ }^{3} \Sigma_{u}^{+}(6 S+6 S)$ potential. The field intensity was $2.5 \mathrm{~kW} / \mathrm{cm}^{2}$. The inset shows the position of the Feshbach resonance on the two dressed potentials (energy of the potentials in $\mathrm{cm}^{-1}$ ). The energy shift $E_{f}$ is $8545 \mathrm{~cm}^{-1}-$ by $3187 \mathrm{~cm}^{-1}$ red-detuned to the $6 S \rightarrow 6 P_{3 / 2}$ transition. 